\title{
Einführung der Eigentumsgarantie in die Verfassung der VR China
}

\author{
Von Wenguang $Y u$, Frankfurt/M. ${ }^{1}$
}

Die neueste Verfassungsänderung der Volksrepublik China, verabschiedet am 14. März 2004, kann man wohl als einen Meilenstein der Entwicklung der chinesischen Verfassung bezeichnen, denn die Gewährleistung des privaten Eigentums ist zum ersten Mal in die Verfassung aufgenommen worden. ${ }^{2}$ Dies erregte nicht nur bei der Bevölkerung in China, sondern auch weltweit große Aufmerksamkeit. ${ }^{3}$ Gleichzeitig stellt sich die Frage, welche praktische Bedeutung die Verankerung des Eigentumsschutzes in der Verfassung für das sozialistische China hat. Welche Hintergründe hat diese Verfassungsnovelle? Werden diese neuen verfassungsrechtlichen Bestimmungen in Zukunft auch wirklich umgesetzt? Welche Probleme könnten auftreten? Im vorliegenden Aufsatz wird zuerst die neuste Verfassungsänderung vorgestellt, dann wird der historisch-politische Kontext der Einführung des Eigentumsschutzes in die Verfassung skizziert. Anschließend wird die praktische Bedeutung der Änderung beleuchtet. Schließlich werden einige Probleme mit der Umsetzung der Eigentumsgarantie in der Praxis und Theorie untersucht.

Die Autorin bedankt sich bei ihrem Doktorvater, Prof. Dr. Ebsen, für seine Vorschläge und Bemerkungen zu diesem Aufsatz.

2

Obwohl der Schutz des Eigentums bereits in der älteren Verfassung (VerfCh von 1954 und Art. 13 a.F. VerfCh von 1982) vorgesehen ist, gab es damals den Begriff „privates Eigentum“ nicht. Gemäß Art. 13 Abs. 1 a.F. VerfCh von 1982 wurde das Recht der Bürger auf Eigentum an ihren erworbenen Einkommen, Ersparnissen, Häusern und anderen legalen Vermögen vom Staat geschützt. Deshalb bezeichnet man es als partielle Eigentumsgarantie wegen des begrenzt geschützten Eigentums. Vgl. Sun, Yafei und Min, Jiaqiao, Zhongguo si chan baohu 26 nian ji (Die Chronik der Eigentumsgarantie in China in den vergangenen 26 Jahren), Nanfang zhoumo (Süd-Wochenende Zeitung), 18. März 2004.

3 z. B.: China codifies Property and Human Rights, Washington Post, 15. 03. 2004, Page A14; China Changes Constitution to address Rights Issues, The New York Times, 14. 03. 2004, in: http://www.nytimes.com/2004/03/14/international/asia/14CND-CHIN.html?ex=1095739200\&en $=\mathrm{db} 114 \mathrm{~d} 16 \mathrm{e} 540 \mathrm{ef0d} \& \mathrm{ei}=5070$; China endorses private Property, BBC NEWS, 15. 03. 2004, in: http://news.bbc.co.uk/2/hi/asia-pacific/3509850.stm; China: Verfassungsänderung auf dem Papier, Frankfurter Allgemeine Zeitung, 19. 03. 2004, Nr.67/Seite 14; Volkskongress verankert Schutz des Privateigentums in Verfassung, Süddeutsche Zeitung, 14. 03. 2004, Siehe: http://www. sueddeutsche.de/ausland/artikel/446/28418/; Les biens privés reconnus par la Constitution, Le FIGARO, 15. 03. 2004. Einzelheiten zu dieser Änderung bei Nicole Schulte-Kulkmann / Lea Shih / Sebastian Heilmann, Änderungen der Verfassung der Volksrepublik China (2004): Übersetzung und Kommentar, VRÜ 37 (2004), S. 345 ff 


\section{Zur Änderung der Verfassungsgarantie des privaten Eigentums ${ }^{4}$}

Im Unterschied zu der umfassenden deutschen Eigentumsgarantie in Art. 14 GG ist der Eigentumsschutz in der chinesischen Verfassung in drei Artikel aufgeteilt: „Schutz des legalen privaten Eigentums“ in Art. 13, „Staatliche Enteignung und Beanspruchung von Grund und Boden“ in Art. 10 und „Die rechtliche- und politische Position der nichtstaatlichen Wirtschaft" in Art. 11. Die Änderung der Verfassung bezüglich der Eigentumsgarantie betrifft diese drei Artikel folgendermaßen:

\section{Einführung der Begriffe „Privates Eigentum “ und „Vermögensrecht“}

Im Zuge der Verfassungsänderung wurden die juristischen Begriffe „, Privates Eigentum“ ${ }^{5}$ und „Vermögensrecht“" ${ }^{6}$ zum ersten Mal in die Verfassung der Volkrepublik China (VerfCh) eingeführt. Dadurch hat der Schutz des privaten Eigentums gegenüber dem Schutz des öffentlichen Eigentums an Bedeutung gewonnen. Nunmehr ist nach der Verfassung nicht nur das öffentliche Eigentum unantastbar (Art. 12 Abs. 1 VerfCh) ${ }^{7}$, sondern auch das private Eigentum (Art. 13. Abs. 1 n.F. VerfCh). Der Begriff ,das Recht auf Eigentum (Eigentumsrecht)“ in der alten Fassung ist in der neuen Fassung durch den Begriff „Vermögensrecht“" ersetzt worden, der klarer und umfassender ist, ${ }^{8}$ weil das Eigentumsrecht im Bereich des chinesischen Zivilrechts normalerweise als das Recht des Eigentümers auf die Gesamtheit von Besitz, Gebrauch, Ertrag und Verfügung eigener Sachen ${ }^{9}$, also als ein dingliches Recht verstanden wird. Das Vermögensrecht dagegen umfasst nicht nur das Sachenrecht, sondern auch Schuldrecht, Urheberrecht, und die anderen das Vermögen betreffenden Rechtssätze. ${ }^{10}$ Die Gewährleistung des „Vermögensrechts“ anstelle des „Eigentumsrechts“ erweitert den Schutzbereich.

Wang, Zhaoguo, Die Erklärung zum Verfassungsänderungsentwurf der Volksrepublik China. Wang, Zhaoguo, der stellvertretende Vorsitzende des Ständigen Ausschusses des Nationalen Volkskongresses, hat am 8. März 2004 diese Erklärung bei der zweiten Tagung des zehnten Nationalen Volkskongresses vorgetragen.

§ 71 Allgemeine Grundsätze des Zivilrechts (verkündete am 12. 4. 1986 und trat am 1. 1. 1987 in Kraft); Zhang, Junhao, Minfa xue yuanli, (Grundlage des Zivilrechts, Aufl. 3 2000), S. 419; Wang, Liming, Minfa (Zivilrecht), 2000, S. 154.

Wang, Liming, a.a.O, S. 157. 


\section{Erweiterung des Schutzbereiches}

Der Schutzbereich der Eigentumsgarantie hat sich auf Produktionsmittel (im Sinne des Begriffs im Marxismus) ausgedehnt. ${ }^{11}$ Früher beschränkte sich die verfassungsrechtliche Eigentumsgewährleistung nur auf Lebenshaltungsmittel, z.B. Einkommen, Ersparnisse, Häuser, usw. (Art. 13 Abs. 1 a.F. VerfCh). Jetzt werden alle legalen privaten Vermögensrechte durch die Verfassung geschützt. (Art. 13 Abs. 1, 2 n.F. VerfCh ).

\section{Einführung der gesetzmäßigen Entschädigung für Enteignung}

Es ist nun eine gesetzmäßige Entschädigung für Enteignung oder Beanspruchung durch den Staat vorgesehen (Art. 13 Abs. 3 n.F. VerfCh). Bei dieser Einführung sind die folgenden beiden Aspekte zu beachten.

Auf der einen Seite unterscheiden die neuen Bestimmungen zum ersten Mal Enteignung und Beanspruchung (Art. 10 Abs. 3 und Art. 13 Abs. 3 n.F.). Nach der überwiegenden Ansicht der chinesischen Juristen wird das Eigentumsrecht durch Enteignung dem Staat übertragen, während sich durch Beanspruchung nur der Inhaber des Nutzungsrechts ändert. ${ }^{12}$ Der Unterschied hat zur Folge, dass der beanspruchte Gegenstand dem früheren Eigentümer zurückzugeben ist, wenn jener zur Befriedigung öffentlicher Interessen nicht mehr benötigt wird, wohingegen bei der Enteignung wegen der Übergabe des Eigentumsrechts die Rückgabe des enteigneten Gegenstandes nicht mehr in Frage kommt. Früher wurden diese beiden unterschiedlichen Rechtsverhältnisse sowohl in der Verfassung als auch im Grund- und Bodenverwaltungsgesetz einheitlich aber uneindeutig mit nur einem Wort, nämlich „Beanspruchung“, bezeichnet.

Auf der anderen Seite wird die Entschädigung für Enteignung oder Beanspruchung betont. Der Staat kann ${ }^{13}$ in Übereinstimmung mit den gesetzlichen Bestimmungen den Betroffenen entschädigen. Besondere Bedeutung hat dies wegen der zur Zeit statt findenden umfangreichen Sanierung der Städte und der Umwandlung von Ackerland in Industriegebiete. Nicht zuletzt spielen die wirtschaftlichen Interessen der Immobilienfirmen bei der Erschließung der Grundstücke eine große Rolle. Es gab in den vergangenen Jahren viele rechtliche Auseinandersetzungen wegen des Abrisses von Häusern in den Städten und der diesbezüglichen Umsiedlung der Bewohner aus den Stadtzentren in Randgebiete sowie wegen Enteignungen von Bauern in ländlichen Gebieten. Das Recht des Eigentümers wurde durch

Wang, Zhaoguo, a.a.O.

13

Wang, Zhaoguo, a.a.O.

Das Problem mit dem Wort „kann“ in diesem Artikel der Verfassung wird unten in diesem Aufsatz erörtert. 
Behörden oder Immobilienfirmen im Namen des so genannten „öffentlichen Interesses“ beeinträchtigt. Manchmal wurden die Häuser oder Grundstücke ohne Entschädigung oder mit unverhältnismäßig niedriger Entschädigung enteignet bzw. beansprucht. Obwohl die Entschädigung schon im Grund- und Bodenverwaltungsgesetz festgelegt worden war ${ }^{14}$, kam es häufig zu Enteignungen ohne entsprechende Entschädigungen. Davor soll die verfassungsrechtliche Eigentumsgarantie nunmehr schützen.

\section{Verbesserung der Rechtsstellung der nichtstaatlichen Wirtschaft}

Die Rechtsstellung der nichtstaatlichen Wirtschaft hat sich in den neuen Vorschriften der Verfassung verbessert, nach denen sie vom Staat zu fördern und zu unterstützen ist (Art. 11 Abs. 2 n.F. VerfCh). Vorher wurde die nichtstaatliche Wirtschaft vom Staat eher überwacht und verwaltet (Art. 11 Abs. 2 a.F. VerfCh). Die Verfassungsänderung steht in engem Zusammenhang mit dem derzeitigen dynamischen Wachstum der privaten Wirtschaft in China.

\section{Einführung der Respektierung und Gewährleistung der Menschenrechte}

Respektierung und Gewährleistung der Menschenrechte werden zum ersten Mal in die Verfassung eingeführt (Art. 33 Abs. 3 n.F. VerfCh). Obwohl der Zusammenhang zwischen der Eigentumsgarantie und den Menschenrechten in dieser Verfassung noch unklar bleibt, ist nicht zu bezweifeln, dass die Eigentumsgarantie als ein in der Allgemeinen Erklärung der Menschrechte verankertes Recht ${ }^{15}$ durch die Vorschrift des Artikel 33 Abs. 3 n.F. VerfCh verstärkt geschützt werden sollte.

Laut § 47 I Grund- und Bodenverwaltungsgesetz, verabschiedet am 25. Juni 1986 und revidiert am 29 August 1998 (Land Administration law of the People's Republic China) „Land requisitioned shall be compensated for on the basis of ist original purpose of use." Gemäß $\$ 58$ II Grundund Bodenverwaltungsgesetz „, The user granted with the land-use right shall be compensated appropriately when ist right to the use of state-owned land i staken back according to the provisions of sub-paragraphs (1) and (2) in the preceding paragraph.", The Law of the People's Republic of China, Band 10 (1998). schaft mit anderem Recht auf Eigentum; niemand darf willkürlich seines Eigentums beraubt werden." 


\section{Hintergründe der Änderung zum Privateigentumsschutz}

\section{Die Rolle der Verfassung in der VR China}

Bevor die Hintergründe der Verfassungsänderung angegangen werden, soll zunächst die Rolle der Verfassung im politischen und rechtlichen System Chinas vorgestellt werden.

Seit ihrer Gründung 1949 wurde in der VR China bereits vier Mal eine Verfassung erlassen (1954, 1975, 1978, 1982). Die bestehende Verfassung aus dem Jahre 1982 wurde bisher vier Mal revidiert, und zwar in den Jahren 1988, 1993, 1999 und 2004. Der häufige Wechsel und die vielen Änderungen der Verfassung hängen eng mit dem Wandel der politischen Linie der Kommunistischen Partei Chinas (KPCh) zusammen. Die Verfassungsänderungen spiegeln den jeweiligen politischen Kurs der Partei wider. Die Vorschläge für die jeweiligen Verfassungsänderungen, die dem chinesischen Parlament, also dem Nationalen Volkskongress (NVK) gemacht wurden, kamen direkt von der KPCh. Die KPCh-Vertreter hielten normalerweise im Oktober, fast ein halbes Jahr vor der Tagung des NVK, einen Nationalen Parteitag ab, bei dem das Zentralkomitee der Partei einen Vorschlag zur Verfassungsänderung verabschiedete, der dann beim nächsten, normalerweise im März stattfindenden NVK eingebracht und von diesem verabschiedet wurde. In diesem Sinne ist die Verfassung „ein juristisches Echo der jeweiligen politischen Linie der Partei“. ${ }^{16}$ Über die staatsstiftende Funktion hinaus fungiert die Verfassung vor allem auch als Ausdrucksmittel der zentralen Elemente des politischen Systems.

Außerdem ist die Verfassung das Grundgesetz des politisch- wirtschaftlichen Systems sowie die Grundlage für die einfachen Gesetze. Laut Art. 5 III VerfCh darf kein Gesetz, keine administrative oder lokale Verordnung oder Vorschrift im Widerspruch zur Verfassung stehen. Mit anderen Worten, die Verfassung steht auf dem höchsten Rang in der Normenhierarchie. Alle Staatsorgane, die Streitkräfte, alle politischen Parteien und gesellschaftlichen Organisationen sowie Betriebe und Institutionen sind an die Verfassung gebunden (Art. 5 IV VerfCh). Die Verfassung kann nur mit der Zustimmung von mehr als zwei Dritteln aller Abgeordneten des NVK geändert werden (Art. 64 Abs. 1 VerfCh). Allerdings gilt die Verfassung nicht als Rechtsgrundlage zur Entscheidung konkreter Fälle durch Gerichte. Nach einer früheren justiziellen Interpretation ${ }^{17}$ des Obersten Volksge-

von Senger, Harro, Einführung in das chinesische Recht, 1994, S. 51.

17 Justizielle Interpretation ist die Auslegung durch die höchsten Organe der Justiz, nämlich durch das Oberste Volksgericht bzw. die Oberste Volksstaatsanwaltschaft. Die Interpretationen des Obersten Volksgerichts gibt es in zwei Formen: Zum einen nimmt das Oberste Volksgericht Stellung zu Fragen eines untergeordneten Gerichts anlässlich eines konkreten Falles. Zum anderen äußert sich das Oberste Volksgericht bisweilen losgelöst von einem konkreten Fall in einem allgemeinen Rechtsanwendungserlass. Vgl.: von Senger, Harro, a.a.O, S. 180. 
richts ${ }^{18}$ dürfen die Normen der Verfassung nicht unmittelbar für die Begründung eines Urteils angewandt werden. In einer jüngeren justiziellen Interpretation vom 13. August 2001 hat das Oberste Volksgericht jedoch einer Klägerin ihren Anspruch auf Schadensersatz aus dem Grundrecht auf Bildung gemäß der Verfassung zugebilligt. ${ }^{19}$ Diese Entscheidung rief eine heftige Diskussion hervor. Viele Juristen sehen dadurch die Einschränkung gelockert, die Verfassung auf konkrete Fälle direkt anzuwenden. ${ }^{20} \mathrm{Da}$ das Fallrecht in China theoretisch nicht anerkannt ist, bleibt es fraglich, ob in Zukunft auch andere Fälle im direkten Rückgriff auf die Verfassung entschieden werden.

\section{Historisch-politischer Kontext der Einführung des Eigentumsschutzes in die Verfassung}

Die Entwicklungsgeschichte des Privateigentumsschutzes in der Volksrepublik China wird einerseits von dem Wirtschaftswandel und der Gesellschaftstransformation sehr stark beeinflusst, andererseits aber auch von der sozialistischen ideologischen Perspektive gegenüber dem Eigentum tief geprägt.

a) Die historische Entwicklung der nichtstaatlichen Wirtschaft und die Änderung ihrer Rechtsstellung in der Verfassung

Der Privateigentumsschutz wurde teilweise schon in der Verfassung von 1954 festgelegt, aber nicht wirklich praktiziert. Seit der sog. Kollektivierung von 1956 war das gesamte Privateigentum in die Kollektivbewirtschaftung einbezogen. Das Gemeinwohl oder das öffentliche Interesse hatte gegenüber dem Privateigentumsschutz den absoluten Vorrang. Das Wort „Privateigentum“ war in der Alltagssprache verschwunden und das Thema in der

Zuigao renmin fayuan gei xinjiang gaoji renmin fayuan de pifu 1955: „guanyu zai xingshi panjueshu zhong buyi zhijie yinyong xianfa zuo lunzui kexing de yiju de pifu" (Die schriftliche Antwort des Obersten Gerichts im Jahre 1955 an das Obergericht Xinjian über die Ungeeignetheit der Verfassungsvorschriften als Rechtsgrund in strafrechtlichem Urteil).

Zuigao renmin fayuan guanyu qinfan shou jiaoyuquan an de fashi 2001 di 25 hao pifu (Schriftliche Antwort des Obersten Volksgerichts auf die Frage zum Fall wegen Eingriffs in das Grundrecht auf Bildung, Fashi. Nr 25. 2001).

20

Huang, Songyou, Xianfa sifa hua jiqi yiyi (die Anwendbarkeit der Verfassung im Prozess und ihre Bedeutung), Renming Fayuan Bao (Die Zeitung der Volksgerichte), 13. August, 2001; Jiangping, He Weifang, u.a., Xianfa sifa hua siren tan (Die Diskussion über die Anwendbarkeit der Verfassung im konkreten Fall, Nanfang Zhoumo (Süd- Wochenendezeitung), 19. September 2001, usw. 
Wissenschaft im Zusammenhang mit dem privaten Vermögen tabuisiert. ${ }^{21}$ „Privatheit“ war in jener Zeit das Synonym von Egoismus und sollte beseitigt werden.

Durch die Einführung der Reform- und Öffnungspolitik seit 1978 hat sich die Situation schrittweise verändert. Die individuelle Wirtschaft, nämlich die Tätigkeit der Einzelgewerbetreibenden, hat allmählich das starre Planwirtschaftssystem durchbrochen, und China wandelt sich von der Planwirtschaft über die „sozialistische Warenwirtschaft mit Plan“ hin zur so genannten ,sozialistischen Marktwirtschaft“ (ab 1993), wie die politischen Mottos für die jeweiligen Entwicklungsphasen lauteten. Im Laufe der Wirtschaftstransformation spielte die nichtstaatliche Wirtschaft eine immer wichtigere Rolle. ${ }^{22}$. Insbesondere regional ist die Privatwirtschaft jetzt von großer Bedeutung und dient als wichtige Quelle für den Staatshaushalt. Sie hat auch viele Arbeitplätze geschaffen und zur Verbesserung der Beschäftigungslage beigetragen. ${ }^{23}$

Allmählich haben diese Erfolge und Leistungen der Privatwirtschaft Eingang in die VerfCh gefunden. Im Jahr 1982 wurden erste Investitionen ausländischer Unternehmen gemäß Art. 18 Abs. 1 VerfCh in der VR China gestattet. ${ }^{24}$ Auch die einheimische individuelle Wirtschaft (Einzelgewerbetreibende) wurde als eine Ergänzung der auf dem Gemeineigentum basierenden sozialistischen Wirtschaft zugelassen. Die legalen individuellen Rechte und Interessen in der individuellen Wirtschaft wurden vom Staat geschützt (Art.11 Abs. 1 VerfCh 1982). Nichtstaatliche Unternehmen mit über acht Angestellten, gesetzlich als Unternehmen der „Privatwirtschaft“ definiert, wurden in jener Zeit zwar von den einzelnen

Vgl. Ji Weidong, Zhongguo xianfa gaige de tujing yu caichanquan wenti (Der Weg der Verfassungsreform und das Eigentumsproblem in China), Dangdai zhongguo yanjiu (Die Forschung über das heutige China), 1999 Heft 3.

22

Nach der Statistik in einem Analysebericht über die Entwicklung der Privatwirtschaft im Jahre 2003 trägt die Privatwirtschaft im weiten Sinne mit $63.5 \%$ und die Privatwirtschaft im engen Sinne mit 48.5\% zum binnenwirtschaftlichen Bruttoprodukt im Jahre 2002 bei. Die Zahl der Privatunternehmen hat sich vom Jahre 1990 bis 2002 um das 24-fache gesteigert. Das Stammkapital der Privatwirtschaft im Jahre 2002 ist um das 58-fache auf einen Betrag von 2.800 Mrd Yuan. gestiegen, in: Projektarbeitsgruppe zum Thema „Entwicklung der Privatwirtschaft“ der allchinesischen Vereinigung der Industriellen und Kaufleute, Analysebericht über die Entwicklung der Privatwirtschaft im Jahre 2003, S.10, S. 18, S. 19. Die Privatwirtschaft im weiten Sinne enthält alle nichtstaatlichen Wirtschaftseinheiten, dazu gehören die inländische Privatwirtschaft (Individuelle Wirtschaft, Privatunternehmen, Kollektivunternehmen), Unternehmen, in die von Bürgern Hongkongs, Macaus und Taiwans investiert wurde und ausländische Unternehmen. Die Privatwirtschaft im engen Sinn schließt die ausländischen Unternehmen aus.

Projekt-Arbeitsgruppe, a.a.O, S. 29ff.

24 Art. 18 Abs. 1 VerfCh: Die Volksrepublik China erlaubt ausländischen Unternehmen, anderen ausländischen Wirtschaftsorganisationen oder ausländischen Individuen, in China zu investieren oder mit chinesischen Betrieben oder anderen chinesischen Wirtschaftsorganisationen in verschiedener Form wirtschaftlich zu kooperieren, sofern dies den gesetzlichen Bestimmungen der Volksrepublik China entspricht. 
Gesetzen sowie Verordnungen ${ }^{25}$ erlaubt, fielen aber nach der legalen Definition nicht in die Kategorie „Individuelle Wirtschaft“ im Sinne der Verfassung und standen deswegen nicht ausdrücklich unter deren Schutz.

Die Verfassungsänderung 1988 fügte in Art. 11 einen neuen (dritten) Absatz ein, nach dem die Privatwirtschaft von der Verfassung geschützt und auch als eine Ergänzung der sozialistischen Wirtschaft des Gemeineigentums bezeichnet wurde. Jedoch durfte die Privatwirtschaft den von den Gesetzen erlaubten Umfang nicht überschreiten (Art. 11 Abs. 3 a.F. VerfCh von 1988) und wurde dadurch eingeschränkt.

Erwähnenswert ist als nächstes auch die Änderung der VerfCh im Jahre 1993, nach der die sog. ,,sozialistische Marktwirtschaft“ nunmehr das Wirtschaftssystem der VR China sein soll (Präambel der VerfCh, geändert am 29. März 1993). Die Spielregeln der Gleichberechtigung und fairen Konkurrenz, die in der Marktwirtschaft gelten sollten und die eine entsprechende Rechtsstellung der Privatwirtschaft erfordern, fehlten jedoch auch nach dieser Änderung noch.

Bei der Verfassungsnovelle im Jahre 1999 schließlich stand Art. 11 VerfCh erneut im Mittelpunkt. Absatz 1 des Art. 11 VerfCh wurde dahingehend geändert, dass die Individual- und Privatwirtschaft sowie andere nicht auf Gemeinschaftseigentum beruhenden Wirtschaftsformen als wichtige Bestandteile der sozialistischen Marktwirtschaft, und nicht wie in der alten Form nur als die Ergänzung der Volkswirtschaft, in der Verfassung anerkannt wurden. Nach dem neuen Absatz 2 des gleichen Artikels schützt der Staat die legalen Rechte und Interessen der Privatwirtschaft.

Zwar hatte die Privatwirtschaft nun den Status eines wichtigen Bestandteils der Volkswirtschaft, war aber gegenüber Wirtschaftsformen in Gemeinschaftseigentum benachteiligt. Letztere galten als die dominierende Kraft der Volkswirtschaft, und ihre Entwicklung und Konsolidierung sollte vom Staat gewährleistet werden (Art. 7 VerfCh), wohingegen die Privatwirtschaft zwar vom Staat ermutigt und unterstützt, aber zugleich gelenkt, überwacht und verwaltet werden sollte (Art. 11 Abs. 2 VerfCh). Dies führte zu folgenden Problemen:

Zum einen erschwerte es die weitere Entwicklung der Privatwirtschaft. Denn in der Konkurrenz mit den staatseigenen Unternehmen hatten die privaten in Ermangelung verfassungsrechtlicher Gleichberechtigung auch in der Praxis keine gleichen Chancen. Die Staatsbetriebe erhielten z.B. enorme Finanzsubventionen in Form von Krediten mit sehr niedrigen Zinsen, was für die Privatunternehmen fast unmöglich war. 
Zum anderen misstrauten die Privatunternehmer wegen der mangelnden Rechtssicherheit der Wirtschaftspolitik des Staates. Sie hatten große Angst vor politischen Kampagnen und Willkürmaßnahmen mit Entzug des Eigentums. Folglich tauchten viele sogenannte „Rotkäppchen-Unternehmen“ auf, die sich als Kollektivunternehmen anmeldeten, tatsächlich aber Privatunternehmen waren. Sobald aber diese Privatunternehmer über ihr Eigentum, das sie in solche Unternehmen investiert hatten, verfügen wollten, begegneten sie großen Hindernissen oder sogar Strafen wegen Unterschlagung oder Aneignung von „kollektivem Eigentum“. Viele komplizierte Rechtsstreitigkeiten waren die Folge. ${ }^{26}$

Außerdem zögerten die Privateigentümer auf Grund des fehlenden Privateigentumsschutzes mit weiteren Investitionen oder verlagerten ihre Investitionen ins Ausland. Teilweise verschwendeten sie das Geld auch maßlos, da sie nicht sicher waren, ob ihnen ihr jetziges Eigentum später noch gehören würde.

Um die Fehlentwicklung der Privatwirtschaft zu korrigieren und die Interessen der Privatunternehmer zu schützen, hat die Interessenvertretung der nichtstaatlichen Wirtschaft, die „Allchinesische Vereinigung der Industriellen und Kaufleute“, seit 1998 dreimal einen Vorschlag zur Verfassungsänderung bei der Nationalen Politischen Konsultativkonferenz $(\mathrm{NPK})^{27}$ eingebracht, um eine Einführung einer Eigentumsgarantie zu erreichen. Im Dezember 2003 wurde dieser Vorschlag vom Zentralkomitee der Kommunistischen Partei während des 16. Parteitags als wertvoll und durchsetzbar bewertet und im März 2004 beim NVK vorgebracht und durch diesen verabschiedet.

26

Die Geschichte von Herrn Chen Jinhong ist ein gutes Beispiel dafür. Er hatte im Jahre 1986 mit eigenem Kapital eine Firma gegründet, und im Jahre 1993 ließ er sie als kollektives Unternehmen wieder bei der zuständigen Behörde registrieren. Im Jahr 1994 wurde er aus seinem Amt des Generalmanagers durch die Stadtkommission für Wirtschaft und Handel entlassen. Er hielt die Entscheidung für einen rechtswidrigen Verwaltungseingriff und erhob eine Anfechtungsklage vor Gericht. Nach 8 Jahren gewann er den Prozess in der letzten Instanz. Anschließen verlangte er Schadenersatz im Höhe von 160 Millionen Yuan. Aber die Klage wurde im Dezember 2003 abgewiesen. Im März 2004 beantragte er aufgrund der neuen Verfassungsbestimmung zur Eigentumsgarantie eine Revision. Ob der Antrag angenommen wird, ist noch nicht entschieden. in: $L i$, Gang, Zhongguo xingzheng diyi an (Die größte Verwaltungsklage in China), Shenzhen tiequ bao, 16. Dezember 2003 und $\mathrm{Li}$, Chaotao, Min gao guan diyi an qi bolan (Die Wendung der grösten Klage des Bürgers gegen den Staat), Xinxi shibao (Informationszeitung), 18. März 2004.

Die Politische Konsultativkonferenz des Chinesischen Volkes ist „eine Organisation der Einheitsfront mit umfassendem repräsentativem Charakter"(Präambel, VVC). Sie besteht aus unterschiedlichen Sozialgruppen, z. B. den acht so genannten demokratischen Parteien, die schon vor der Gründung der VR China gegründet wurden und nach der Gründung als "vertraute Zusammenarbeitspartner der kommunistischen Partei" an der Politik teilnehmen, der Gewerkschaft, dem Frauenverband, dem Jungendverband, usw. Die Konferenz tagt einmal pro Jahr genau drei Tage vor der Tagung des nationalen Volkskongresses. Ihre Aufgabe ist es, die Arbeit der Kommunistischen Partei und der Regierung zu beaufsichtigen und konstruktiv zu kritisieren. 
Diese Entwicklungsgeschichte der nichtstaatlichen Wirtschaft und die Geschichte der Einführung des Eigentumsschutzes zeigt, dass sich in der Partei nach und nach die Einstellung zur Eigentumsordnung, die ursprünglich auf der im Marxismus-Leninismus verankerten Doktrin des Gemeineigentums basierte, geändert hat und man dem Wesen der marktwirtschaftlichen Eigentumsordnung näher kommt.

b) Wunsch der Bürger nach Eigentumsschutz

Die Eigentumsgarantie wurde nicht nur von Unternehmern der nichtstaatlichen Wirtschaft gewünscht, die verlangten, dass ihre legalen Rechte und Interessen besser geschützt und ihre Rechtsposition in der Verfassung gestärkt werden sollte, sondern auch von den Bürgern, die seit der Reform und Öffnung immer mehr privates Vermögen erworben hatten, und die diese Einzelinteressen selbstverständlich mit der Eigentumsgarantie durch die Verfassung gesichert wissen möchten. ${ }^{28}$

c) Erfordernisse der Marktwirtschaft

Ein weiterer Grund für die Einführung der Eigentumsgarantie ist, dass Marktwirtschaft und diese wurde ja in Form der sog. "sozialistischen Marktwirtschaft" schon 1993 eingeführt - den Schutz des Eigentums fordert. Die Verkehrsfreiheit der Marktwirtschaft erstreckt sich auf Waren, Personen, Dienstleistungen und Kapital. Eine klare Zuordnung des Eigentums verschiedener Inhaber ist die Voraussetzung für den fließenden Umlauf solcher Faktoren in der Marktwirtschaft. Eigentumsrechte sind ein wichtiger Eckpfeiler der Marktwirtschaft. Derzeit orientiert sich China an der sog. "sozialistischen Marktwirtschaft", aber es fehlt an einer entsprechenden Eigentumsordnung. In Bezug auf das Volkseigentumssystem liegt das Eigentumsrecht an den Produktionsmitteln theoretisch beim Volk, mit anderen Worten, bei allen Bürgern, aber in der Praxis hat ein Bürger keine direkte Verfügung über das Gemeineigentum, diese liegt vielmehr in den Händen von Staatsorganen (Komitees zur Verwaltung von staatlichem Eigentum: guoyou zichan guanli weiyuanhui). ${ }^{29}$ Solches Eigentum besteht überwiegend in Form von Produktionsmitteln in staatlichen Unternehmen. In Ermangelung eines effektiven Kontrollsystems, insbesondere bei der Privatisierung staatlicher Unternehmen, bereichern sich daran aber häufig korrupte Beamte

Laut einer Statistik besitzt die Bevölkerung 57\% des Kapitals im ganzen Land, in: Fan Gang, Zhongguo caifu baogao, (Bericht über Reichtum Chinas), 2003; Nach einer anderen Statistik betrugen die Ersparnisse der Bevölkerung im Jahre 2003 11.069,5 Mrd. Yuan (nach dem damaligen Kurs ca.1100 Mrd Euro), d.h. sie sind im Vergleich zum Jahre 2002 um 17.4\% gestiegen, in: Das Statistikamt der Volksrepublik China, Zhonghua renmin gongheguo 2003 nian guomin jingji he shehui fazhang gongbao (Das statistische Bulletin über die Volkswirtschaft und die Sozialentwicklung im Jahre 2003), 26. Februar 2004.

29

Xiao, Liang, Sichan Baohu Yiyi Zhong (Die große Bedeutung der Eigentumsgarantie), Zhongguo jingji shibao, 30. Dezember 2003. 
oder Mitglieder der Chefetagen solcher Unternehmen. In solchen Situationen ist es oft unklar, welches Eigentum bzw. wie viel davon noch dem Staat gehört. Der enorme Abfluss von Staatseigentum ist ein offenes Geheimnis. Auch daher kam der Vorschlag, ein Gesetz zum Schutz von Staatseigentum zu erlassen, und eine Privateigentumsgarantie in die Verfassung aufzunehmen, um so öffentliches Eigentum von privatem Eigentum scharf abzugrenzen und effektiv zu schützen. ${ }^{30}$

\section{Praktische Bedeutungen der Einführung der Eigentumsgarantie für China}

Steht die neu eingeführte Eigentumsgarantie nur auf dem Papier oder ist sie für die Bürger, die Wirtschaft und die Gesellschaft wirklich von Bedeutung?

\section{Bedeutung für den Einzelnen}

Dass der veränderte Eigentumsschutz der Bevölkerung durchaus bewusst ist, zeigte sich gleich nach der Verabschiedung der neusten Verfassungsänderung: Es gab zahlreiche Fälle, in denen Bürger versucht haben, auf der Grundlage der Verfassung ihre Häuser vor Zwangsabriss zu schützen oder auf höhere Entschädigung zu klagen. ${ }^{31}$

Es ist jedoch zu betonen, dass diese Fälle nur für ein verstärktes Rechtsbewusstsein der Bevölkerung stehen, nicht aber für praktischen erfolgreichen Rechtsschutz. Denn die Verfassung wird als das Manifest der Grundrechte angesehen und besitzt keine Abwehrfunktion für Bürger zum Schutz vor staatlichen Eingriffen. Der Bürger kann nicht direkt anhand der Verfassung, sondern nur mithilfe des einfachen Gesetzes seine konkreten Rechte geltend machen. Deswegen sollten einfache Gesetze bezüglich des Eigentumsschutzes baldmöglichst verabschiedet und existierende verbessert werden.

30

31

Li, Shuguang, jiakuai zhiding guoyou zichan fa (Beschleunigung der Gesetzgebung zum Staatseigentum), Zhongguo jingji shibao, 28. August 2003.

z.B. demonstrierten 38 Einwohner in der Stadt Kaifeng, Provinz Henan, auf der Straße für die Verfassung, insbesondere für die Eigentumsgarantie, um gegen den Abriss ihrer Häuser zu protestieren. Han, Junjie, Henan Kaifeng 38 hu jumin jietou xuanchuan xianfa dizhi chaiqian (38 Einwohner der Stadt Kaifeng, Provinz Henan, demonstrieren für die Verfassung und gegen den willkürlichen Abriss von Häusern und Umsiedlungen.), Zhongguo Qingnian Bao (Chinesische Jugendzeitung), 29. April 2004. Ein Geschäftsmann in Yancheng der Provinz Jiangsu hat eine Klage gegen die Regierung wegen der Streitigkeit über die Höhe der Entschädigung mit Hilfe des Artikels 13 VerfCh „Schutz des privaten Eigentums“" erhoben, in: http://news.sina.com.cn/s/200404-27/00353168490.shtml. 


\section{Bedeutung für die nichtstaatliche Wirtschaft}

Für die private Wirtschaft bedeuten die Eigentumsgarantie und die Anerkennung ihrer Rechtsstellung in der Verfassung bessere Entwicklungschancen und Konkurrenzrahmenbedingungen. Die Tür vieler Wirtschaftsbranchen öffnet sich nunmehr für die Privatwirtschaft. Privatunternehmer können in viele Märkte eintreten, die früher einem staatlichen Monopol unterlagen. So wird beispielsweise die Zusammenarbeit zwischen öffentlicher Hand und den Privaten (Public Private Partnership) auf dem Gebiet der Stadtinfrastruktur wie Wasserversorgung, Abwasserbeseitigung, Abfallentsorgung, etc. gefördert. ${ }^{32}$ Der Eintritt in die neuen Märkte wird durch die Vereinfachung der komplizierten Verwaltungsgenehmigungsverfahren erleichtert. ${ }^{33}$ Das ist eine neue zukunftsträchtige Wirtschaftspolitik, und die Verfassungsänderung ist dafür ein wichtiges Zeichen. Außerdem brauchen die Privatunternehmen durch die Eigentumsgarantie sich nicht mehr als „Rotkäppchen-Unternehmen“, d.h. Kollektivunternehmen, zu tarnen. Die Privatunternehmer können als echte Eigentümer über ihr Vermögen verfügen und es vor staatlichem Eingriff schützen. Sie können in ihre Unternehmen re-investieren und diese langfristig betreiben. Schließlich wird die Privatwirtschaft in der Volkswirtschaft nicht mehr diskriminiert, sie gewinnt stattdessen die gleiche Stellung wie staatliche und ausländische Unternehmen.

\section{Bedeutung für das Wirtschaftsystem}

Auch für das Wirtschaftssystem ist die Einführung der Eigentumsgewährleistung von großer Bedeutung. China befindet sich zur Zeit in der Übergangsphase von der Planwirtschaft zur Marktwirtschaft. Rechtssicherheit ist hierfür eine Voraussetzung und insbesondere im Zusammenhang mit dem WTO-Beitritt auch ein Faktor im Standortwettbewerb. Je besser das Eigentum geschützt wird, desto sicherer und zugleich attraktiver ist China für ausländische Investoren.

Projektarbeitsgruppe, Analysebericht über die Entwicklung der Privatwirtschaft im Jahre 2003, S. 23ff, a.a.O.; Am 28. Dezember 2002 hat das chinesische Bauministerium eine Rechtsverordnung zur Marktöffnung der öffentlichen Infrastruktur verabschiedet. Dabei spielt PPP eine große Rolle; Am 1. Oktober 2003 trat die Vorschrift zum Konzessionsbetrieb in der Stadtinfrastruktur Pekings in Kraft. In dieser Vorschrift wird die Beteiligung der Privatwirtschaft an der Stadtinfrastruktur gefördert. Zukunft der privaten Unternehmen Chinas), Shang Jie (Zeitung für Handelsbereich), 19. Dezember 2003 . 


\section{Probleme mit der Umsetzung der Eigentumsgarantie}

\section{Veränderung oder Erlass von Gesetzen zur Umsetzung des verfassungsrechtlichen Eigentumsschutzes}

Da die Verfassung den Eigentumsschutz nur als Grundsatz vorgeschrieben hat, bedarf es eines Rechtssystems, das aus den unterschiedlichen einfachen Gesetzen besteht und der Durchsetzung der verfassungsrechtlichen Eigentumsgewährleistung dienlich ist. ${ }^{34}$ Deshalb sollen die diesbezüglichen Gesetze entsprechend korrigiert oder neue verabschiedet werden. Laut Gesetzgebungsplan (ein Teil des Fünfjahresplans) des Ständigen Ausschusses des zehnten Nationalen Volkskongresses sollen zahlreiche Gesetzentwürfe in dieser Legislaturperiode (2003-2008) dem NVK oder dessen Ständigem Ausschuss ${ }^{35}$ zur Überprüfung und Beschlussfassung übergeben werden. Es handelt sich um Gesetze zum Sachenrecht, Deliktsrecht, Verwaltungskostenrecht, Verwaltungszwangsvollstreckungsrecht sowie das Staatsvermögensgesetz, Sozialversicherungsgesetz, Arbeitsvertragsgesetz, Gesetz zum Schutz der Rechte und Interessen der Bauern, und Grund- und Bodenverwaltungsgesetz sowie Staatshaftungsgesetz. ${ }^{36}$

Das Sachenrechtsgesetz wird als das wichtigste Gesetz zur Konkretisierung der verfassungsrechtlichen Eigentumsgarantie angesehen; der Gesetzentwurf dazu wurde bis jetzt schon dreimal im Ständigen Ausschuss des NVK diskutiert. Am 10. Juli 2005 hat der Ständige Ausschuss den Entwurf veröffentlicht, um Vorschläge und Anregungen aller Seiten einzuholen. Der Gesetzentwurf umfasst Regelungen zu Grundsätzen und Prinzipien, Eigen-

Cai, Dingjian, zitiert in: Min, Jiaqiao / Sun,Yafei, sichan baohu shi daguo xingqi zhi lu (Der Schutz des Privateigentums ist der Weg zum Aufschwung eines großen Landes), Nanfang zhoumo, 18. März 2004.

Gemäß Art. 62. Nr.3 VerfCh arbeitet der Nationalen Volkskongress die grundlegenden Gesetze über Strafsachen, zivile Angelegenheiten, die Staatsorgane und andere Angelegenheiten aus oder ändert diese ab. Gemäß Art. 67 Nr. 2 hat der Ständige Ausschuss des Nationalen Volkskongresses die Befugnis, die Gesetze mit Ausnahme derer, die vom Nationalen Volkskongress ausgearbeitet werden sollen, auszuarbeiten und abzuändern. Die Abgrenzung von grundlegendem Gesetz und anderem Gesetz sowohl in der Verfassung als auch in dem Gesetzgebungsgesetz ist jedoch nicht klar, somit bleibt es hier offen, welche oben genannte Gesetzentwürfe dem Nationalen Volkskongress übergeben werden sollen und welche dem Ständigen Ausschuss des Nationalen Volkskongresses.

Das Gesetzgebungsvorhaben des Ständigen Ausschusses des zehnten Nationalen Volkskongresses, in: http://news.xinhuanet.com/zhengfu/2004-01/09/content_1268128.htm. Laut dem Vorhaben werden insgesamt 59 Gesetzesentwürfe dem Nationalen Volkskongress oder dem Ständigen Ausschuss des Nationalen Volkskongress zur Überprüfung und Beschlussfassung übergeben werden. Nach der Meinung der Autorin betreffen die oben genannten Gesetzesentwürfe oder Gesetzesänderungen den Eigentumsschutz. 
tum, Nutzungsrecht, Sicherungssachenrecht und Besitz. ${ }^{37}$ Der Schutz des Privateigentums findet seinen Ausdruck in diesem Entwurf. Die konkreten Fragen zum Entwurf im Zusammenhang mit Bodennutzungsrechten werden unten unter Punkt IV 3 (b) dargestellt.

\section{Auslegung der Begriffe „Eigentum“ und „Vermögensrecht“}

In der chinesischen Verfassung existieren keine gesetzlichen Definitionen des Eigentums und des Vermögensrechts. Zwar ist im deutschen Grundgesetz „Eigentum“ auch nicht definiert, aber durch das gesamte einfache Recht und die Rechtsprechung des Bundesverfassungsgerichts ist das verfassungsrechtliche Eigentum und dessen Schutzbereich definiert und ausgelegt worden ${ }^{38}$.

Doch gibt es in der Volksrepublik China weder ein Verfassungsgericht noch ein anderes für den Einzelfall eingerichtetes Verfassungsschutzorgan. Zwar hat der Ständige Ausschuss des Nationalen Volkskongresses gemäß Art. 67 Nr. 1 VerfCh die Befugnis, die Verfassung allgemein auszulegen, aber er hat diese Macht bisher noch nie ausgeübt, geschweige denn auf die konkrete Beschwerde eines Bürgers hin. Daher haben die Bürger keine Möglichkeit, wegen eines Eingriffs in ein Grundrecht, z.B. in das Eigentum, Rechtsschutz direkt bei einem obersten Staatsorgan zu erlangen. So bleibt den Bürgern für den Schutz ihres Eigentums nur die Klage vor den einfachen Gerichten. Wie oben schon erwähnt, ist es aber innerhalb chinesischer Juristenkreise noch sehr umstritten, ob die Verfassungsvorschriften direkt und in konkreten Fällen vor einfachen Gerichten angewendet werden dürfen. Daher ist es schwer möglich, dass der Begriff des Eigentums und sein Schutzbereich in China durch die Gerichte ausgelegt werden.

\section{Schutzbereich der Eigentumsgarantie}

In Bezug auf den Umfang der Eigentumsgarantie stellt sich die Frage, welche Rechte unter dem neuen hinzugefügten Begriff „Vermögensrecht“ verstanden werden können bzw. welche konkreten Rechte dem verfassungsrechtlichen Vermögensrecht zugeordnet werden können. Es ist auch offen, was die Maßstäbe für diese Zuordnung sind und ob öffentlichrechtliche Ansprüche, z.B. sozialversicherungsrechtliche Ansprüche, auch dazu gehören. Im Folgenden werden die Sozialversicherungsansprüche, der Grundbesitz auf dem Land

Zhonghuarenmingongheguo wuquanfa caoan quanwen [Sachenrechtsgesetz der Volksrepublik China (Entwurf)], in: http://npc.people.com.cn/GB/14957/3530629.html. Deutsche Übersetzung siehe: http://www.gtz-legal-reform.org.cn/uploaden/forumdisplay.php?fid=3. 
und das Wohnungseigentumsrecht in Städten exemplarisch für die Bestimmung des Schutzbereiches thematisiert.

\section{a) Sozialversicherungsansprüche}

Unter den früheren Bedingungen der Planwirtschaft in der Volksrepublik China wurde das Existenzminimum der Bürger von ihren Arbeitseinheiten in den Städten bzw. ihren Volkskommunen auf dem Land gewährleistet. Während des Übergangs von der Planwirtschaft zur Marktwirtschaft ist diese Gewährleistung stark geschwächt worden, und ein allgemeines Sozialversicherungssystem ist zur Zeit in China noch nicht eingerichtet. Dies gefährdet die Stabilität der chinesischen Gesellschaft, insbesondere wenn man an die Zunahme der Arbeitslosigkeit und Landflucht sowie die fortschreitende Überalterung der Gesellschaft denkt. Die Einrichtung eines Sozialversicherungssystems ${ }^{39}$ wurde mittlerweile in der chinesischen Verfassung festgelegt, aber die Frage, ob die Ansprüche aus der Sozialversicherung unter das Vermögensrecht fallen, ist noch zu klären.

Beispielsweise wird die chinesische Krankenversicherung über Gemeinschaftsfonds und Individualkonten finanziert. Der Arbeitgeber zahlt monatlich ca. 6\% der gesamten Lohnsumme des jeweiligen Betriebs als Beiträge. Diese Beiträge werden wiederum so aufgeteilt, dass ca. $70 \%$ dem Gemeinschaftsfonds und 30\% den Individualkonten zufließen. Die Beiträge der Arbeitnehmer betragen 2\% ihres Monatslohns und fließen komplett auf das Individualkonto. ${ }^{40}$ Die Einlage und der Zins des Individualkontos gehören dem Versicherten, sie können ausschließlich vom Versicherten genutzt, abgeschlossen und vererbt werden. Die Gemeinschaftsfonds werden dagegen vom Sozialversicherungsträger einheitlich verwaltet und umverteilt und die Versicherten können darüber nicht direkt verfügen. Aus den Gemeinschaftsfonds werden nur die Kosten des Krankenhausaufenthaltes ausgezahlt, und sie werden regional nach Kreisen bzw. Städten getrennt verwaltet. Wenn ein Arbeitnehmer in eine andere Stadt umzieht und dort arbeitet, behält er sein Individualkonto. Die Beiträge, die sein ehemaliger Arbeitgeber in den Gemeinschaftsfonds eingezahlt hat, bleiben aber im Gemeinschaftsfonds der ursprünglichen Stadt. Die Leistungsniveaus der Fonds sind von Stadt zu Stadt unterschiedlich und es ist ungeklärt, ob der Versicherte einen

Art. 14. Abs. 4, VerfCh: Der Staat richtet ein Sozialversicherungssystem ein, das dem Niveau der wirtschaftlichen Entwicklung entspricht, und vervollständigt es.

40

Siehe: Guowuyuan guanyu jianli chengzhen zhigong jiben yiliao baoxian zhidu de jueding (Beschluss des Staatsrates zur Errichtung einer Basiskrankenversicherungsordnung für die städtischen und kleinstädtischen Beschäftigten vom Dezember 1998); Vgl.: Rösner, Hans Jürgen, Das chinesische Krankenversicherungssystem in der Transformationsphase: Erste Bewertung und Politikvorschläge, in: Internationale Revue für Soziale Sicherheit, 57, 3/2004 S. 83 ff; Darimont, Barbara, Sozialversicherungsrecht der V.R. China, 2004; Hanisch, Holger / Obst, Andreas, Auswirkung der wirtschaftlichen und militärischen Reformen Chinas auf das chinesische Sozialsystem, in: ZIAS 2003, S. 59 ff. 
Rechtsanspruch auf das Leistungsniveau der Stadt hat, in der er vor dem Umzug gelebt hat. Die bestehenden sozialrechtlichen Bestimmungen bieten keine ausdrückliche Antwort auf die Frage, welche vermögenswerten Rechtsansprüche auf Krankenversicherung bzw. andere Sozialversicherungen zum Vermögensrecht i.S.d. Art. 13 VerfCh gehören. Es besteht noch keine entsprechende Änderung bezüglich des Eigentumsschutzes im Sozialrecht. Im Zuge der Einrichtung eines Sozialversicherungssystems bedarf es hier einer eindeutigen Klärung.

$\mathrm{Ob}$ andere öffentlich-rechtliche Rechtspositionen (Ansprüche und Anwartschaften auf Renten aus der Sozialversicherung und Anwartschaften auf Arbeitslosengeld) unter den Schutzbereich des Eigentums fallen, ist auch zu klären. In Deutschland hat das Bundsverfassungsgericht die oben genannten Rechtspositionen dem Eigentum im Sinne Art. 14 GG zugeordnet ${ }^{41}$. Nach dem Zweck und der Funktion der Eigentumsgarantie unter Berücksichtigung ihrer Bedeutung im Gesamtgefüge der Verfassung bestimmt sich die rechtliche Einordnung einer vermögenswerten Rechtsposition als im Art.14 GG geschütztes Eigentum. ${ }^{42}$ Diese Maßstäbe für den Schutzbereich der Eigentumsgarantie können für China ein wertvoller Hinweis sein. So ist es zu hoffen, dass die Eigentumsgarantie in der VerfCh, ähnlich wie in Deutschland, auch den Anspruch auf Sozialversicherung einschließt.

b) Vermögensrechte auf ländlichen Grund und Boden

Ein weiteres kompliziertes Thema im Zusammenhang mit der Eigentumsgarantie ist der Grundbesitz. In China gehört der Boden entweder zum Staats- oder zum Kollektiveigentum, aber nicht zum Privateigentum. Gemäß Art.10 VerfCh ist der Boden in den Städten Staatseigentum, und der Boden auf dem Lande und in den Vororten der Städte Kollektiveigentum, mit Ausnahme der Teile, die gemäß gesetzlichen Bestimmungen auch dem Staat gehören. Grundstücke und Parzellen zur privaten Nutzung auf Acker- und Bergland sind ebenfalls Kollektiveigentum.

Der Boden bzw. das Grundstück darf weder ganz noch teilweise im Sinne des Eigentumsrechts verkauft, belastet oder unterverpachtet werden. Seit der Verfassungsänderung im Jahre 1988 darf nur das sog. Bodennutzungsrecht vom Staat oder von den Kollektiveigentümern gesetzmäßig abgetreten werden. ${ }^{43}$

Der Boden auf dem Land als Kollektiveigentum verursacht höchste Unklarheit und Unbestimmtheit der Eigentumsverhältnisse. Zuerst ist es unklar, wer der tatsächliche Eigentümer

Wendt, in: Sachs (Hrsg.), Grundgesetz-Kommentar, 3. Aufl., 2000, Art. 14, Rdn. 34.

42

Wendt, a.a.O, Rdn. 22.

43

Art. 10 Abs. 4 Satz 2 VerfCh: Das Bodennutzungsrecht kann gemäß den gesetzlichen Bestimmungen abgetreten werden. 
des ländlichen Bodens ist. Nach Verfassung und Gesetz gehört der ländliche Boden dem Kollektiv der Bauern (Art. 10 VerfCh, § 74 Abs. 2 Satz 1 Allgemeine Grundsätze des Zivilrechts und $\S 10$ Grund- und Bodenverwaltungsgesetz). Aber das Bauernkollektiv ist eine völlig abstrakte Einheit und keine juristische Person. Wie kann es dann als Eigentümer seine Rechte ausüben und schützen? Das Kollektivmitglied, also der einzelne Bauer, kann das Eigentum am Boden auch nicht besitzen. Deshalb ist der Eigentümer des ländlichen Bodens eine Scheinexistenz. Das hat zur Folge, dass die Nutzungsrechte, Verfügungsrechte und Ertragsrechte auf den Boden nicht effektiv geschützt werden können.

Wie schon erwähnt, besitzen die Bauern das von ihnen bewirtschaftete Land nicht als Eigentum, sie haben keinen Eigentumstitel darauf. Sie können dieses Land weder kaufen noch verkaufen. Allerdings haben sie Nutzungsrechte auf den Boden, die sich in vertraglich festgelegten Bewirtschaftungsrechten verkörpern. Laut Gesetz wird der ländliche Boden von der Organisation der ländlichen Kollektivwirtschaft oder vom Dorfkomitee bewirtschaftet oder verwaltet ( $\$ 74$ Abs. 2 Satz 1 Allgemeine Grundsätze des Zivilrechts und § 10 Grund- und Bodenverwaltungsgesetz). Die erstere Organisation ist eine abstrakte Einheit, die letztere ein Selbstverwaltungsorgan. Beide sind keine juristischen Personen ( $\$ 2$ Organisationsgesetz des Dorfkomitees; § 2, 10, 14 Grund- und Bodenverwaltungsgesetz), haben jedoch die Befugnis, mit den jeweiligen Bauernfamilien einen Bodennutzungs- und Betriebsvertrag abzuschließen $(\S 12$ Gesetz zum Vertrag über Bewirtschaftung des ländlichen Bodens, verabschiedet am 29 August 2002). Diese Bewirtschaftungsrechte können durch Vertrag übertragen, unterverpachtet, ausgetauscht oder überlassen werden und können innerhalb der jeweiligen Bauernfamilien vererbt werden. ( $§ 10,16,32$ Das Gesetz zum Vertrag über Bewirtschaftung des ländlichen Bodens).

Diese Nutzungsrechte auf dem Boden in China ähneln der sog. ,,Verleihung von Nutzungsrechten an volkseigenen Grundstücken“ der ehemaligen DDR, womit in der DDR die Möglichkeit zur Aufspaltung von Eigentum an und Nutzung von Grund und Boden geschaffen worden war ( $\$ 287-291$ ZGB). ${ }^{44}$ (In Grenzen) Vergleichbares gibt es in der Bundesrepublik mit Erbbaurecht und Wohnungseigentumsrecht.

Im Unterschied zu diesen eindeutigen dinglichen Nutzungsrechten für Grundstücke in Deutschland bestehen viele Probleme mit den Nutzungsrechten des genossenschaftlichen Bodens in China.

Es ist zunächst zu fragen, welche Rechtsnatur die Bewirtschaftungsrechte besitzen. Sind sie dingliche oder vertragliche Nutzungsrechte? Die bestehenden Gesetze bieten keine zufriedenstellende Antwort. Die Rechtsverhältnisse im Vertrag über die Bewirtschaftungsrechte 
sind ebenfalls kompliziert. Dabei sind die Nutzungsrechte der Bauernfamilien, das Vertragsrecht zwischen Bauernfamilien und Dorfkomitee und die Verwaltungsaufgabe des Dorfkomitees miteinander vermengt. Der Sachenrechtsgesetzentwurf hat die durch Vertrag festgelegten Bewirtschaftungsrechte als Sachenrecht geregelt und die Übertragbarkeit der Bewirtschaftungsrechte des ländlichen Bodens bestätigt und ausführlich geregelt. ${ }^{45}$.

Weitere Fragen sind: Wie kann über die Nutzungsrechte tatsächlich störungsfrei verfügt werden? Gehören solche Rechte zum Vermögensrecht und wie können sie durch eine verfassungsrechtliche Eigentumsgarantie geschützt werden?

In der Praxis werden die Nutzungsrechte bisweilen beschränkt oder sogar beeinträchtigt. So können z.B. die Bauern den Nutzungszweck des Bodens nicht selbst bestimmen, und der Boden kann nicht anders als landwirtschaftlich bewirtschaftet werden ( $\$ 17$ Gesetz zum Vertrag über Bewirtschaftung des ländlichen Grund und Bodens). Wenn die ganze Bauernfamilie in die Stadt umzieht, kann sie die Bewirtschaftungsrechte nicht an Dritte übertragen, sondern muss auf die Rechte verzichten ( $\$ 26$ Gesetz zum Vertrag über Bewirtschaftung des ländlichen Grund und Bodens).

Aus gesetzlicher und theoretischer Sicht fallen die Nutzungsrechte selbstverständlich unter das Vermögensrecht und sollten geschützt werden. Aber die Rechte werden häufig durch administrative Enteignung oder durch vom Leiter des Dorfkomitees verhandelte rechtswidrige Übertragung des Bodennutzungsrechts beeinträchtigt. Wegen der Urbanisierung und Industrialisierung wurde sehr viel Ackerland durch den Staat entzogen, aber die Inhaber der Nutzungsrechte haben keine Möglichkeit, auf die Höhe der Entschädigung und das Verfahren der Entschädigung Einfluss zu nehmen. Die Regierung und das Dorfkomitee fällen die Entscheidung. Die Entschädigung ist folglich sehr niedrig. Außerdem wird es häufig von Leitern des Dorfkomitees ausgenutzt, dass der Eigentümer des ländlichen Bodens nicht eindeutig festgelegt ist. Obwohl es gemäß gesetzlichen Bestimmungen nur mit Zustimmung von mehr als zwei Dritteln der Bauernvertreter in einem Dorf getan werden darf $(\$ 15$ Grund- und Bodenverwaltungsgesetz), treten sie das Bodennutzungsrecht ohne Mitteilung oder Zustimmung der betroffenen Bauern an Dritte (in den meisten Fällen an Immobilienhändler und im Namen des öffentlichen Interesses) ab, um sich so zu bereichern. Die Bauern verlieren dadurch das Bodennutzungsrecht sowie ihre Lebensgrundlage. ${ }^{46}$ Dadurch kommt es zu vielen Enteignungsstreitigkeiten. ${ }^{47}$. In diesem Fall können die Bauern gegen das Dorfkomitee klagen, aber die Rechtsnatur der Klage (zivilrechtliche oder verwaltungs-

Siehe $\S \S 132,133,134$ Sachenrechtsgesetzentwurf (Dritter Diskussionsentwurf).

Cheng Jie, Guanzhu tudi zhengyong zhong de caichanquan baozhang (Aufmerksamkeit gegenüber dem Eigentumsschutz während der Bodenbeanspruchung), Fazhi ribao (Legaldaily), 30. 
rechtliche) ist sehr umstritten, weil einerseits das Dorfkomitee als Selbstverwaltungsorgan zwar keine Behörde ist, es aber andererseits auch Verwaltungsaufgaben ausführt. ${ }^{48}$ Außerdem besteht zwischen dem Dorfkomitee und den Bauern ein Bodenbewirtschaftungsvertrag.

Alle oben dargestellten schwer lösbaren Fragen können auf die Bodenordnung zurückgeführt werden. Boden als wichtiges Produktionsmittel muss nicht zwingend im Privateigentum stehen. Öffentliches Eigentum heute oder, historisch gesehen, die von einer Dorfgemeinschaft gemeinsam genutzte Allmende ${ }^{49}$ seien hier Beispiele. Aber angesichts der Unklarheit der Rechtsverhältnisse des genossenschaftlichen Bodens in China ist es offensichtlich, dass ohne eine Reform der Kollektiveigentumsordnung des Bodens auf dem Land ein angemessener Eigentumsschutz für private Rechte an Grund und Boden nicht zu gewährleisten ist. Viele chinesische Wissenschaftler haben auf diese Problematik aufmerksam gemacht. Es wurden ganz unterschiedliche Reformvorschläge zum Eigentumssystem für Grundstücke auf dem Land gemacht: z.B. Privatisierung (Grund und Boden sei Privateigentum, die Bauer seien Eigentümer des ländlichen Bodens) ${ }^{50}$, Verstaatlichung (Kollektiveigentum solle abgeschafft werden, aller Grund und Boden sei Staatseigentum, die Bauer würden mit dem Staat einen Nutzungsvertrag schließen) ${ }^{51}$, Miteigentum nach Anteilen (jeder Bauer hätte Eigentum an dem Boden, den er besitzt) ${ }^{52}$, oder eine Mischung aus Volkseigentum, Kollektiveigentum und Privateigentum (jetziges Eigentumssystem bliebe, hinzu käme das Privateigentum, je nach der Möglichkeit und dem Erfordernis) ${ }^{53}$. Unter Berücksichtigung des bestehenden sozialistischen politischen und wirtschaftlichen Systems in China scheint ein Miteigentum nach Anteilen am sinnvollsten. Jedenfalls ist es dringend geboten, das Problem des Grundbesitzes und die Theorie der Nutzungsrechte auf Boden aus Sicht des Sachenrechts gründlich zu erforschen und dann den Schutz der Bodennut-

Zuo, Zhiping / Chen, yin, Shenli nongcun jiti jingji zuzhi shouyi fenpei jiufen anjian zhong wenti zhi qianxi (Einfache Analyse der Probleme in den Streitigkeiten über die Ertragsverteilung der ländlichen kollektiven Wirtschaftsorganisationen), in: http://www.law-lib.com/lw/lw_view.asp?no $=3793$.

Zückert, Hartmut, Allmende und Allmendaufhebung, 2003; Lutz, Christiane, Meilensteine der Allmend-Entwicklung in Bayern, in: http://www.ilpoe.uni-stuttgart.de/projekte/allmende/Publikas/geschichte_lutz.htm.

Yang, Xiaokai, Zhongguo tudi siyouhua de yiyi (Die Bedeutung der Privatisierung des Grunds und Bodens in China), in: http://www.yannan.cn/data/detail.php?id=425.

51 Wang, Weiguo, zitiert in: Tudi daodi suan shuide? (Wem gehört das Grundstück?), Xinwen zhoukan, 29. Dezember 2003.

Han, Jun, Minque jieding nongmin de tudi caichan quanli, qianghua guojia dui nongmin tudi chanquan de baohu. (Die Eigentumsrechte an Grund und Boden der Bauern müssen eindeutig festgelegt und der staatliche Schutz verstärkt werden), Zhongguo jingji shibao, 22. Juli 2003. 
zungsrechte in dem Sachenrechtsgesetzentwurf festzulegen. Der zur Zeit diskutierte Sachenrechtsgesetzentwurf räumt ein, dass das vertraglich festgelegte Bewirtschaftungsrecht übertragen, ausgetauscht, unterverpachtet oder überlassen werden darf. Grund und Boden auf dem Land als Kollektiveigentum bleibt aber unberührt [§§ 61, 62 Sachenrechtsgesetzentwurf (Dritter Diskussionsentwurf)].

c) Wohnungseigentumsrecht in den Städten

Nach der Verfassung ist der Boden in den Städten Staatseigentum. Mittlerweile haben sehr viele Stadtbewohner ihre eigenen Häuser gekauft, aber Eigentümer eines Hauses haben nur Nutzungsrecht am Boden, dieses Nutzungsrecht beträgt maximal 70 Jahre. ${ }^{54}$ Diese Befristung des Nutzungsrechts verursacht die Unsicherheit des Wohnungseigentumsrechts, d. h. es ist unklar, ob die Wohnung nach dem Ablauf der Frist auch an den Staat übergeht oder ob der Wohnungseigentümer das Recht hat, das Nutzungsrecht am Boden zu verlängern. Falls dies der Fall wäre, stellte sich die Frage nach der Dauer der Verlängerung und nach dem Preis für das verlängerte Nutzungsrecht. Diese Unsicherheit ist sowohl die große Sorge der Wohnungseigentümer in den Städten als auch ein erhebliches Investitionshemmnis für Immobilienanleger. Der Sachenrechtsgesetzentwurf räumt ein, dass der Inhaber des Nutzungsrechts am Boden dieses unter folgenden Voraussetzungen verlängern kann: Er muss ein Jahr vor Fristablauf die Verlängerung beantragen und es darf dem kein öffentliches Interesse entgegenstehen. Der Inhaber des Eigentumsrechts muss dem Antrag zustimmen. Nach der Verlängerung muss der Berechtigte den vereinbarten Veräußerungspreis zahlen ( $\$ 155$ Sachenrechtsgesetzentwurf). Aber es wurden in diesem Entwurf längst nicht alle Fragen beantwortet, insbesondere falls das Nutzungsrecht z. B. aufgrund des sog. „Öffentlichen Interesses“ nicht verlängert wird: Wem gehört dann das Haus auf dem Boden? Wie kann das Wohnungseigentumsrecht in diesem Fall geschützt werden?

\section{Voraussetzung der Enteignung und Entschädigung}

Nach Art. 10 Abs. 3 und Art. 13 Abs. 3 VerfCh kann der Staat Grund und Boden oder das private Eigentum der Bürger enteignen oder beanspruchen und die Betroffenen entschädigen, wenn es die öffentlichen Interessen erfordern.

Diese Vorschriften werfen zwei Gruppen von Problemen auf: rechts an staatlichem Boden in den Städten und Kreise (verabschiedet am 19 Mai 1990) darf das Nutzungsrecht am Boden zum Wohnen maximal 70 Jahre übertragen werden. 
Einerseits ist das "öffentliche Interesse" ein unbestimmter Rechtsbegriff und bedarf der Klärung. Wer kann sich auf das öffentliche Interesse berufen? (Es steht wohl außer Zweifel, dass die Interessen von Immobilienfirmen auf keinen Fall dazu gehören sollten). Auch ist unklar, wie das Ausmaß der Entschädigung zu gestalten ist - verhältnismäßig oder voll? Dieses Problem ist sehr wichtig, denn die meisten Streitigkeiten gab es wegen der Höhe der Entschädigung. Drittens sind die Verfahren für Enteignung noch nicht gesetzlich geregelt. Soll z.B. der Betroffene das Recht auf Anhörung während einer Bodennutzungsplanungsphase haben? Inwieweit trägt das Eigentum außer der Enteignung im öffentlichen Interesse andere Sozialpflichten? Wie solche Pflichten in konkreten Entscheidungen umgesetzt werden können, ist ebenfalls unklar.

Andererseits lauten die o.g. Vorschriften der VerfCh, dass der Staat die Betroffenen entschädigen „kann“. Es scheint, zumindest nach dem Wortlaut dieser Vorschriften, dass der Staat nicht entschädigungspflichtig ist, sondern freien Spielraum hat. Wenn dieses „kann“ auf Entscheidungsermessen des Staats hindeuten würde, wäre es mit dem „Rechtsstaatsprinzip“ (Art. 5 Abs. 1 VerfCh) ${ }^{55}$ nicht vereinbar, weil die Entschädigungspflicht für Enteignung oder Beeinträchtigungen des Eigentums durch den Staat das wesentliche Element der Rechtstaatlichkeit ist. Nach der Wortlautauslegung ist diese Bestimmung offen zu verstehen, aber nach dem Sinn und Zweck kann man sie vielleicht so auslegen, dass der Staat Eigentum enteignen kann und dann entschädigen muss.

Es ist zu betonen, dass im Sachenrechtsgesetzentwurf (Dritter Diskussionsentwurf) großer Wert auf die Problematik der Enteignung und Entschädigung gelegt wurde. ${ }^{56}$ Nach dem Entwurf muss der Enteignete angemessen entschädigt werden und diejenigen, die wider-

Das „Rechtsstaatsprinzip“ wurde im Jahr 1999 in der chinesischen Verfassung verankert. Art. 5 Abs. 1 VerfCh lautet: „The People's Republic of China governs the country according to law and makes it a socialist country ruled by law.“,. In: The Laws of the People's Republic of China, Band 13 (1999). Schon der Wortlaut des Art. 5 Abs. 1 VerfCh macht deutlich, dass das chinesische Rechtsstaatsprinzip nicht identisch mit dem deutschen ist. Allerdings wird das Prinzip in China nach dem Vorbild Deutschlands rechtswissenschaftlich ausgelegt. Vgl. Jiang, Minan (Hrsg.), Verwaltungsrecht und Verwaltungsprozessrecht, 2000, S. $43 \mathrm{ff}$.

$\S 68$ Sachenrechtsgesetzentwurf schreibt vor: „Der Staat schützt das Eigentum Privater. Es ist verboten, unter dem Namen des Abrisses oder der Enteignung etc. ungesetzlich die Rechtszugehörigkeit des Vermögens Privater zu verändern. Wenn unbewegliches Vermögen Privater abgerissen oder enteignet wird, muss gemäß den staatlichen Bestimmungen eine Entschädigung gewährt werden; gibt es keine staatlichen Bestimmungen, muss eine angemessene Entschädigung gewährt werden; es muss sichergestellt werden, dass diejenigen, deren unbewegliche Sachen abgerissen oder enteignet werden, in geeigneter Weise untergebracht werden. Wenn einem Privaten infolge eines widerrechtlichen Abrisses oder einer widerrechtlichen Enteignung ein Vermögensschaden entsteht, so muss die zivilrechtliche und verwaltungsrechtliche Verantwortung getragen werden; handelt es sich um eine Straftat, wird sie nach dem Gesetz strafrechtlich verfolgt." Vgl. Deutsche Übersetzung des Sachenrechtsgesetzentwurfs von GTZ, in: http://www.gtz-legal-reform.org.cn/ uploaden/forumdisplay.php?fid=3. 
rechtliche Abriss- oder Enteignungsmaßnahmen ergreifen und Vermögensschaden verursachen, müssen die zivilrechtliche, verwaltungsrechtliche und sogar strafrechtliche Verantwortung tragen. Der Sachenrechtsgesetzentwurf stellt eine positive Entwicklung dar trotzdem fehlen noch konkrete Bestimmungen zum Enteignungs- und Entschädigungsverfahren.

\section{5. $\quad$ Prüfung der Verfassungsmäßigkeit von Rechtsnormen in Bezug auf die Eigentumsgarantie}

Eine weitere Frage bei der Umsetzung der verfassungsrechtlichen Eigentumsgarantie ist, wie man die Verfassungsmäßigkeit eines einfachen Gesetzes oder einer untergesetzlichen Rechtsnorm bezüglich des Eigentumsschutzes prüfen kann.

Laut Art. 5 III VerfCh besitzt die Verfassung den höchsten Rang in der Normenhierarchie. Alle Staatsorgane sind an die Verfassung gebunden (Art. 5 IV VerfCh). Aber ohne die Möglichkeit einer Überprüfung bleibt der Vorrang der Verfassung nur ein politischer Appell an den NVK. Während das Bundesverfassungsgericht in Deutschland die Befugnis hat, die Verfassungsmäßigkeit des Eigentumsschutzes in einfachen Gesetzen zu überprüfen, fehlt in China ein effektives System der Normenkontrolle. Zwar hat der Ständige Ausschuss des Nationalen Volkskongresses (SAS) die Normenkontrollbefugnis (Art. 67 Nr. 7, 8 VerfCh; $\S 90,91$ Gesetzgebungsgesetz (verabschiedet am 15. März, 2000)), aber diese Befugnis beschränkt sich nur auf Verordnungen, Vorschriften, Entscheidungen und Anordnungen des Staatsrats sowie die lokalen Verordnungen, Vorschriften und Beschlüsse der Organe auf Provinzebene (Art. 67 Nr. 7, 8 VerfCh), d.h. die Verfassungsmäßigkeit der Gesetze wird nicht vom SAS überprüft. Außerdem hat der SAS diese Befugnis noch nie benutzt, d.h. bislang wurde noch keine Rechtsnorm wegen Verfassungswidrigkeit für nichtig erklärt. ${ }^{57}$ Nicht zuletzt birgt die Vereinigung von legislativen wie judikativen Aufgaben in der Hand eines einzigen Organs, das dazu eigentlich ausschließlich der Legislative zugeordnet sein sollte, erhebliche Gefahren. Es ist somit anzunehmen, dass die Normenkontrolle am Maßstab der Verfassung in absehbarer Zeit in China nicht effektiv gewährleistet sein wird, was bedeutet, dass trotz der Eigentumsgarantie durch die Verfassung, Verletzungen des Eigentumsrechts noch nicht völlig ausgeschlossen werden können, insbesondere wenn sie durch Gesetze und untergesetzliche Vorschriften geschehen.

Um dieses Problem zu lösen, haben chinesische Juristen verschiedene Vorschläge gemacht, z.B. ein Verfassungsgericht nach dem Vorbild des deutschen Verfassungsgerichts oder ein 
Verfassungsrat wie in Frankreich, das aber im Nationalen Volkskongress einzurichten sei. Eine weitere Überlegung ist die Befugnis der Überprüfung der Verfassungsmäßigkeit durch das oberste Volksgericht. ${ }^{58}$

Die neueste bemerkenswerte Entwicklung ist die Errichtung eines Normenkontroll- und Protokollbüros im Rechtsausschuss auf Arbeitsebene des Ständigen Ausschusses des Nationalen Volkskongresses im Mai 2004. ${ }^{59}$ Seine Aufgabe ist es, die Verfassungsmäßigkeit der untergesetzlichen Normen zu überprüfen und Protokolle über untergesetzliche Normen für die Archive und evtl. weitere Arbeiten des Ständigen Ausschusses des Volkskongresses anzufertigen. Dieses Büro ist jedoch, wie der Name schon sagt, nur eine Einrichtung auf Arbeitsebene. Es hat daher keine Befugnis, die Entscheidung über die Verfassungsmäßigkeit einer Norm zu treffen, sondern kann nur seine Feststellungen über verfassungswidrige Normen an die speziellen Ausschüsse des Nationalen Volkskongresses weiterleiten, die diese Normen noch einmal überprüfen, und sie danach dem Ständigen Ausschuss vorlegen können. Der Ständige Ausschuss wiederum kann dann eine endgültige Entscheidung treffen.

Es zeigen sich bei dieser Verfahrensweise zwei Schwächen: Die eine ist der sehr komplizierte Prüfungsablauf und die andere der enge Umfang der zu überprüfenden Normen. Es werden nämlich nur untergesetzliche Vorschriften auf ihre Verfassungsmäßigkeit geprüft. Gesetze gehören aber noch nicht dazu. ${ }^{60}$ Deswegen gilt das Büro nur als ein Übergangsorgan für die Normenkontrolle der Verfassungsmäßigkeit.

In Zukunft soll ein Verfassungskomitee beim Nationalen Volkskongress eingerichtet werden. $^{61}$ Aber auch in diesem Falle könnte die Effektivität der Normenkontrolle nicht vollständig gewährleistet sein, weil letztendlich das gesetzgebende Organ über sich selbst wachen würde.

Laut $\S 90$ Abs. 2 des Gesetzgebungsgesetzes kann ein Bürger dem Ständigen Ausschuss des Volkskongresses einen Hinweis auf die Verfassungswidrigkeit einer untergesetzlichen Norm geben. In Bezug auf den Eigentumsschutz haben 116 Bürger am 14. Juli 2003 vorgeschlagen, dass der Ständige Ausschuss des Volkskongresses die Verfassungsmäßigkeit der „Verordnung des Staatsrats zum Abriss der Häuser und damit verbundene Umsiedlungen“

Jiang, Ping / He, Weifang, Xianfa sifahua siren tan (Die Diskussion über die Anwendbarkeit der Verfassung im konkreten Fall), Nanfang zhoumo, 13. Septemer 2001. ersten Spezialorgan für Überprüfung der Verfassungswidrigkeit), Xin Jing Bao (Xin JingZeitung), 19. Juni 2004.

61

Jiang, Minan, in Liao, Weihua, a.a.O.

Jiang, Minan, in Liao, Weihua, a.a.O. 
und der „Vorschrift zum Abriss der Häuser und damit verbundene Umsiedlungen der Stadt Hangzhou" überprüfen solle. ${ }^{62}$ Die Entscheidung ist jedoch bis jetzt noch nicht getroffen. Bemerkungswert ist aber, dass die Kanzlei des Staatsrats am 6. Juni 2004 eine Verwaltungsvorschrift mit dem Titel „Mitteilung der Kanzlei des Staatsrats über die Kontrolle und striktere Verwaltung des Ausmaßes des Abrisses von Häusern und damit verbundene Umsiedlungen " verabschiedet hat. ${ }^{63}$ In dieser Verwaltungsvorschrift werden die lokalen Regierungen auf allen Ebenen aufgefordert, sich von den gewerblichen Gesellschaften für den Abriss der Häuser zu trennen, und es werden alle gewaltsamen Zwangsmaßnahmen zum Abriss streng verboten. Die Betroffenen sollen vor dem Abriss ihrer Häuser ausreichend Schadensersatz erhalten. ${ }^{64}$ Der Erlass dieser Verwaltungsvorschrift ist vermutlich von jener Eingabe der Bürger beeinflusst worden.

Obwohl der Vorschlag der Bürger zur Normenkontrolle der Verfassungsmäßigkeit vermutlich in gewisser Weise ein Echo gehabt hat, haben die Bürger aber nicht das Recht, ein Verfahren der Verfassungsmäßigkeitsprüfung in Gang zu setzen.

\section{Zusammenhang mit den anderen Grundrechten}

Neben den bisher diskutierten Fragen zur Eigentumsgarantie selbst ist noch die Frage zu untersuchen, inwieweit die übliche Eigentumsgewährleistung ein Grundrecht insbesondere im Sinne der Allgemeinen Erklärung der Menschenrechte (AEMR) ist. Diese stellt fest, dass jeder Mensch allein oder in Gemeinschaft mit anderen ein Recht auf Eigentum hat und niemand willkürlich seines Eigentums beraubt werden darf. Nach Meinung der deutschen Juristen ist die Eigentumsgarantie im Ursprung ein Freiheitsrecht ${ }^{65}$, ein elementares Grundrecht $^{66}$, eines der klassischen Menschenrechte ${ }^{67}$. ,Bürgerliche und politische Freiheit

Wei, Wenbiao, Cong chaiqian tiaoli kan weixian shengcha (Die Beobachtung der Überprüfung der Verfassungswidrigkeit im Blick auf die Verordnung für den Abriss von Häusern und Umsiedlungen), Jingji cankao bao (Informationszeitung zur Wirtschaft), 3. September 2003; Zhuming de weixian shengcha jianyi (Einige relevante Ratschläge zur Überprüfung der Verfassungswidrigkeit) shenyang jinbao (Die Shenyanger Zeitung zum Heute), 14. Januar 2004.

Guowuyuan bangongting di 46 hao wen: ,guowuyuan guanyu kongzhi chengzhen fangwu chaiqian guimo, yange chaiqian guanli de tongzhi“ (,Mitteilung der Kanzlei des Staatsrats über die Kontrolle und striktere Verwaltung des Ausmaßes des Abrisses von Häusern und damit verbundenen Umsiedlungen", GFB (2004) Nr. 46 von der Kanzlei des Staatrats am 6. Juni 2004).

Zhao, Bo, Guowuyuan jiaoting yeman chaiqian, chaiqin gongsi bixu yu zhengfu tuogou (Der Staatsrat fordert die lokalen Regierungen auf allen Ebenen auf, sich von den gewerblichen Gesellschaften für den Abriss von Häusern zu trennen, und verbietet die brutale Maßnahmen des Abrisses von Häusern und damit verbundenen Umsiedlungen.), Beijing yule xinbao, 15. Juni 2004.

66

Badura, a.a.O, S. 10.

BVerfGE, 42, (76/77). 
bleiben ohne eine auf Privatautonomie gegründete Eigentumsordnung und ohne den Schutz des Privateigentums auch an den Produktionsmitteln wesenlos. “68

Schon die Systematik der VerfCh zeigt, dass der Status der Eigentumsgarantie in China nicht so eindeutig ist.

Bei der Änderung der VerfCh 2004 werden der Eigentumsschutz im ersten Kapitel „Allgemeine Grundsätze“ und die Menschenrechte im zweiten Kapitel „Grundrechte und -pflichten der Bürger" normiert. Einerseits kann dies darauf hindeuten, dass die Bedeutung der Eigentumsgarantie betont wird. Andererseits kann es auch ein Mangel der Verfassungsstruktur sein, weil es zwischen den beiden Kapiteln keinen systematischen Zusammenhang gibt. So ist es nicht klar, wie sich der Eigentumsschutz in Art. 13 mit dem Menschenrechtsschutz in Art. 33 verbindet. Auch könnte eine Höherbewertung der - schließlich grundlegenden - Menschenrechte wünschenswert sein. Ungeachtet dieser Problematik ist diese Veränderung der VerfCh jedoch ein sehr großer Fortschritt des Menschenrechtsschutzes in der chinesischen Rechtsgeschichte und zwar wegen beider Einfügungen: der des Eigentumsschutzes und der des Menschenrechtsschutzes.

\section{Schlussbemerkung}

Ausgelöst vom Wirtschaftswandel und der Gesellschaftstransformation, hat der Eigentumsschutz nunmehr entsprechend unumkehrbar Eingang in die chinesische Verfassung gefunden. Das ist ein großer Fortschritt in der chinesischen Verfassungsentwicklung. Aber ein langer Weg steht China noch bevor, denn es sind noch viele Fragen offen: Von der Auslegung der Begriffe Eigentum bzw. Vermögensrecht, über den Schutzbereich der Eigentumsgarantie (z. B, Sozialversicherungsansprüche und die Grundbesitzproblematik), die Prüfung der Verfassungsmäßigkeit von Rechtsnormen in Bezug auf die Eigentumsgarantie, das Ausmaß und das Verfahren der Entschädigung, bis hin zum Zusammenhang des Eigentumsschutzes mit anderen Grundrechten. In China ist nicht nur Eigentumsschutz, sondern auch die Verteilung des sozialen Reichtums von wachsender Bedeutung. Neben dem Eigentumsschutz sind eine gerechte Verteilung des Reichtums und ein Sozialversicherungssystem auf die Tagsordnung der politischen Diskussion gekommen, was beides Reformen der politischen und gesellschaftlichen Ordnung erfordert. ${ }^{69}$

Sieckmann, Jan-Renard, Zum verfassungsrechtlichen Eigentumsschutz im deutschen und britischen Recht, 1999, S. 15.

68

Badura, a.a.O, S. 10.

69

Ji, Weidong, a.a.O. 


\section{Anhang}

Art.10. Abs. 3 a.F. VerfCh 1999: Der Staat kann in Übereinstimmung mit den gesetzlichen Bestimmungen Grund und Boden beanspruchen, wenn dies öffentliche Interessen erfordern.

Neue Fassung der VerfCh 2004: Der Staat kann in Übereinstimmung mit den gesetzlichen Bestimmungen Grund und Boden enteignen oder beanspruchen und den Betroffenen entschädigen, wenn dies öffentliche Interessen erfordern.

(宪法第十条第三款

“国家为了公共利益的需要，可以依照法律规定对土地实行征用。” 修改为：“国家为了公共利益的需要, 可以依照法律规定对土地实行征收或者征用并 给予补偿。”)

Art. 11. Abs. 2 a.F. Der Staat schützt die legalen Rechte und Interessen der individuellen Wirtschaft und privaten Wirtschaft. Der Staat lenkt, überwacht und verwaltet die individuelle Wirtschaft und die private Wirtschaft.

Neue Fassung der VerfCh 2004: Der Staat schützt die legalen Rechte und Interessen der nicht -staatlichen Wirtschaft, wie der individuellen Wirtschaft und privaten Wirtschaft. Der Staat fördert, unterstützt und lenkt die Entwicklung der nicht-staatlichen Wirtschaft und überwacht und verwaltet sie.

(宪法第十一条第二款“国家保护个体经济、私营经济的合法的权利和利益。国家对 个体经济、私营经济实行引导、监督和管理。”

修改为：“国家保护个体经济、私营经济等非公有制经济的合法的权利和利益。国家 鼓励、支持和引导非公有制经济的发展，并对非公有制经济依法实行监督和管理”)

Art. 13.a.F. VerfCh: Der Staat schützt das Recht der Bürger auf Eigentum an ihren legal erworbenen Einkommen, Ersparnissen, Häusern und anderem legalen Vermögen. Der Staat schützt gemäß den gesetzlichen Bestimmungen das Recht der Bürger auf Erbschaft von Privatvermögen.

Neue Fassung der VerfCh 2004: Das legale private Eigentum ist unantastbar. Der Staat schützt gemäß den gesetzlichen Bestimmungen das private Vermögens- und Erbrecht des Bürgers. Der Staat kann in Übereinstimmung mit den gesetzlichen Bestimmungen das private Eigentum des Bürgers enteignen oder beanspruchen und Betroffene entschädigen, wenn dies öffentliche Interessen erfordern.

(宪法第十三条“国家保护公民的合法的收入、储蓄、房屋和其他合法财产的所有权” “国家依照法律规定保护公民的私有财产的继承权。” 
修改为：“公民的合法的私有财产不受侵犯。”“国家依照法律规定保护公民的私有财 产权和继承权。”“国家为了公共利益的需要, 可以依照法律规定对公民的私有财产 实行征收或者征用并给予补偿。”)

Neue Fassung des Art. 14. Abs. 4 der VerfCh 2004: Der Staat richtet ein Sozialversicherungssystem ein, das dem Niveau der wirtschaftlichen Entwicklung entspricht und vervollständigt es.

(宪法第十四条增加一款, 作为第四款 : “国家建立健全同经济发展水平相适应的社 会保障制度。”)

Neue Fassung des Art. 33. Abs. 3. der VerfCh 2004: Der Staat respektiert und gewährleistet die Menschenrechte.

(宪法第三十三条增加一款，作为第三款 : “国家尊重和保障人权。”) 